Biorheology Vol. 15, p. 418.

Pergamon Press Ltd. 1978. Printed in Great Britain

THE THIRD

\title{
INTERNATIONAL CONGRESS OF BIORHEOLOGY
}

\author{
HONORARY CHAIRMAN: ROy Swank \\ CHAIRMAN: Yuan-Cheng Fung \\ SECRETARY GENERAL: John G. Pinto \\ TREASURER: Savio Woo \\ TECHNICAL EXHIBITION: Larry Malcom \\ FINANCE: Sidney Sobin \\ SOCIAL PROGRAM: Luna Fung \\ LOCAL ARRANGEMENTS: Michael Yen, Chairman \\ Eugene Mead, Paul Zupkas, Nancy Bingham, \\ Debbie Yager, Peter Chen, Randy Leonard, \\ Taka Nakagawa, Charles Chuong
}

